\title{
Consumo de psicotrópicos em meio a pandemia do Sars-CoV-2
}

\author{
Consumption of psychotropics among the Sars-CoV-2 pandemic
}

\author{
Fernanda Pinto Dantas Oliveira ${ }^{\circ}$, Fernando Maia Pereira Santos ${ }^{\circ}$, Bruna Dallaqua" $\bullet$ \\ Universidade Salvador (UNIFACS), Salvador, Bahia, Brasil. *Autor para correspondência. E-mail: bruna.jaquie@unifacs.br
}

\begin{abstract}
Resumo: Introdução: A síndrome respiratória causada pelo novo Coronavírus provocou diversas alterações na vida da população mundial, houve a necessidade de adequação e alteração daquilo que era até então compreendido como "normal". A falta de bem-estar mental desencadeou diversos efeitos negativos no cotidiano da população, como transtornos, distúrbios do sono e sofrimento psíquico. Faz-se necessário abordar a medicalização da população, um dos principais mecanismos utilizados para superar as repercussões psicológicas em um cenário de pandemia como o da covid-19. Desta maneira, o objetivo deste estudo é de identificar se a pandemia do novo Coronavírus possibilitou um maior uso de psicotrópicos durante o período de isolamento. Revisão: Foi conduzida uma busca nas bases de dados da Biblioteca Virtual em Saúde (BVS), Medline, LILACS, Scientific Eletronic Library Online (Scielo) e a Biblioteca do Instituto Albert Einstein, utilizando palavras-chave de acordo com o objetivo do artigo. Discussão: Autores apontam uma maior correlação entre a pandemia e o desenvolvimento de transtornos como ansiedade e depressão entre jovens e jovens adultos, aliado a isto, destacaram-se o aumento na venda de medicamentos como antidepressivos, ansiolíticos e substâncias como álcool e drogas ilícitas. Considerações finais: O presente estudo aponta para um aumento do uso de fármacos psicotrópicos entre jovens e adultos, definindo uma correlação entre o aumento de transtornos como depressão e ansiedade e o uso de substâncias como forma de minimizar impactos causados pela pandemia. Porém, reforça-se a necessidade de mais estudos como este, sendo cruciais para identificação e verificação do consumo de medicamentos e outras substâncias nesse período entre jovens, e tornando-se mais uma ferramenta de gestão contra as repercussões causadas pelo coronavírus.
\end{abstract}

Palavras-chave: adulto jovem, ansiedade, COVID 19, depressão, saúde mental.

\begin{abstract}
Introduction: The respiratory syndrome caused by the new Coronavirus caused several changes in the life of the world population, there was a need to adapt and change what was until then understood as "normal". The lack of mental well-being triggered several negative effects on the daily life of the population, such as disorders, sleep disorders and psychological distress. It is necessary to address the medicalization of the population, one of the main mechanisms used to overcome psychological repercussions in a pandemic scenario such as that of covid-19. Thus, the aim of this study is to identify whether the pandemic of the new Coronavirus enabled a greater use of psychotropic during the period of isolation. Review: A search was conducted in the databases of the Virtual Health Library (VHL), Medline, LILACS, Scientific Eletronic Library Online (Scielo) and the Library of the Albert Einstein Institute, using keywords according to the purpose of the article. Discussion: Authors point out a greater correlation between the pandemic and the development of disorders such as anxiety and depression among young people and young adults, in addition to this, the increase in the sale of drugs such as antidepressants, anxiolytics and substances such as alcohol and illicit drugs stood out. Final considerations: The present study points to an increase in the use of psychotropic drugs among young people and adults, defining a correlation between the increase in disorders such as depression and anxiety and the use of substances as a way to minimize impacts caused by the pandemic. However, the need for more studies like this is reinforced, being crucial for the identification and verification of the consumption of drugs and other substances in this period among young people, and becoming another management tool against the repercussions caused by the coronavirus.
\end{abstract}

Keywords: young adult, anxiety, COVID-19, depression, mental health.

\section{Introdução}

A síndrome respiratória causada pelo novo Coronavírus (SARS-CoV-2) foi inicialmente detectada em dezembro de 2019 na cidade de Wuhan na China e desde 11 de março de 2020 foi classificada como uma pandemia, termo que se refere à distribuição geográfica de uma doença (em vários países e regiões do mundo), 
pela Organização Mundial da Saúde (OMS), atingindo vários indivíduos de diferentes classes socioeconômicas e causando uma mudança no cenário mundial, sendo considerada a maior pandemia do século (CDC COVID19 Response Team, 2020; Faro et al., 2020; WHO, 2020; Velavan \& Meyer, 2020).

Para além das mudanças clínicas e epidemiológicas, o padrão comportamental da população foi drasticamente alterado e desta forma, famílias não apenas do Brasil, mas de diversos países se viram incapazes de seguir com suas atividades externas, fossem elas relacionadas a lazer ou trabalho, iniciando um período de isolamento social. Houve a necessidade de adequação e alteração daquilo que era até então compreendido como "normal". Além da quarentena que foi fundamental para que ocorresse uma desaceleração da propagação do vírus, protegendo contra a infecção aqueles com maior risco de quadros graves e reduzindo o pico de necessidade por assistência médica em hospitais e UTI. Ao passar dos meses, o uso de máscaras nos ambientes e espaços se tornaram medidas obrigatórias e entrou na rotina dos brasileiros (Bahia, 2020; Caetano et al., 2020; Faro et al., 2020). Desta forma, o novo normal tem exigido das famílias brasileiras esforços que ocasionam situações de stress, fadiga e ansiedades devido ao grau de incerteza e instabilidade que o vírus proporcionou no país corroborando para um aumento de doenças que afetam a saúde mental (Silva et al., 2020).

Todo o contexto dos fatos, notícias, noticiários e relatos da doença ao redor do mundo, além de sua alta propagação contribuíram para um aumento da ansiedade e depressão entre a população brasileira com relação ao aumento da incidência da doença no país, criando um estado de "Coronafobia", movido pelo aumento estado de alerta adotado pela sociedade (Barros et al., 2020; Duarte et al., 2020; Huang \& Zhao, 2020).

A falta de bem-estar mental desencadeia diversos efeitos negativos no cotidiano da população, como transtornos, distúrbios do sono e sofrimento psíquico, gerando um percentual de mais de $40 \%$ de jovens com diagnósticos relacionados a depressão e ansiedade durante a pandemia, principalmente durante o isolamento social. A pandemia do novo Coronavírus também provocou altos níveis de estresse tanto pelo medo de contrair a doença como pela insegurança em padrões sociais e econômicos (Fontes \& Neri 2015; Barros et al., 2020).

Sob esta ótica, faz-se necessário abordar a medicalização da população, um dos principais mecanismos utilizados para superar as repercussões psicológicas em um cenário de pandemia como o da Covid-19. Desta maneira, o objetivo deste estudo foi identificar se a pandemia gerada pelo SARS-CoV-2 influenciou no uso de fármacos psicotrópicos durante o período de isolamento pela população.

\section{Revisão}

Para realização do presente estudo, foi conduzida uma revisão de literatura a respeito da utilização de medicamentos ansiolíticos e antidepressivos, drogas lícitas e ilícitas para minimizar os efeitos do isolamento social e da pandemia na saúde mental da população. Para isto, foi realizada a busca de artigos nas bases de dados da Biblioteca Virtual em Saúde (BVS), Medline, LILACS, Scientific Eletronic Library Online (Scielo) e a Biblioteca do Instituto Albert Einstein, através do uso dos descritores: pandemia, coronavírus, COVID19, saúde mental, depressão e psicotrópicos, utilizando-se também operadores booleanos como AND e OR para a melhor obtenção dos resultados. Por se tratar de um tema recente, não houve limitação de tempo para a busca dos artigos. Foram incluídos artigos de produção original, em língua inglesa e portuguesa, excluindo outras revisões e artigos de opinião. Também foram excluídos artigos que impossibilitaram o acesso livre e gratuito a seu conteúdo.

Quadro 1. Perfil de artigos encontrados nas bases de dados eletrônicas.

\begin{tabular}{|c|c|c|c|}
\hline Título & Principais Resultados & Periódicos & Autor/Ano \\
\hline $\begin{array}{c}\text { Epidemia de COVID-19 na China e } \\
\text { problemas psicológicos associados }\end{array}$ & $\begin{array}{c}\text { O estudo mostra maior frequência de } \\
\text { ansiedade, depressão e uso prejudicial de álcool } \\
\text { principalmente na faixa entre 21-40 anos. }\end{array}$ & Asian J Psychiatr. & Ahmed et al., 2020 \\
\hline $\begin{array}{c}\text { Uso de substâncias, saúde mental e } \\
\text { comportamentos relacionados ao peso } \\
\text { durante a pandemia de COVID-19 em } \\
\text { pessoas com obesidade }\end{array}$ & $\begin{array}{c}\text { Dos 589 entrevistados, aproximadamente } 24 \% \\
\text { relataram uso de opióides, 10\% de sedativos e } \\
3,5 \% \text { de cannabis e 1\% de estimulantes. Além } \\
\text { de agravamento de sintomas de ansiedade, } \\
\text { tristeza e dificuldade para dormir. }\end{array}$ & Clinical Obesity & Almandoz et al., \\
2021 \\
\hline $\begin{array}{c}\text { Saúde mental, uso de substâncias e } \\
\text { ideação suicida durante a pandemia } \\
\text { COVID-19 - Estados Unidos, } 24 \text { a 30 de } \\
\text { junho de 2020 }\end{array}$ & $\begin{array}{c}\text { Adultos jovens, minorias e diversos } \\
\text { saúde mental, aumento do uso de substâncias } \\
\text { psicotrópicas (álcool e cigarro) e aumento da } \\
\text { intenção suicida. }\end{array}$ & $\begin{array}{c}\text { Morbidity and } \\
\text { mortality weekly } \\
\text { report }\end{array}$ & Czeisler et al., 2020 \\
\hline
\end{tabular}




\begin{tabular}{|c|c|c|c|}
\hline $\begin{array}{l}\text { Como é o uso de substâncias por } \\
\text { adolescentes durante a pandemia de } \\
\text { COVID-19? Examinando mudanças na } \\
\text { frequência, contextos sociais e } \\
\text { preditores relacionados à pandemia }\end{array}$ & $\begin{array}{l}\text { Frequência aumentada do uso de álcool e } \\
\text { Cannabis entre jovens, desde o uso em } \\
\text { momentos de isolamento ( } 49,3 \%) \text {, com amigos } \\
\text { por chamada de vídeo ( } 31,6 \%) \text { e até mesmo em } \\
\text { encontros pessoalmente com amigos ( } 23,6 \%) \text {. }\end{array}$ & J Adolesc. Health & Dumas et al., 2020. \\
\hline $\begin{array}{l}\text { Compreendendo o impacto da } \\
\text { pandemia COVID-19 no estresse, } \\
\text { humor e uso de substâncias entre } \\
\text { jovens adultos na grande Minneapolis- } \\
\text { St. Área de Paul: Resultados do projeto } \\
\text { EAT }\end{array}$ & $\begin{array}{l}\text { Dos } 670 \text { participantes, } 84 \% \text { relataram que a } \\
\text { pandemia influenciou de alguma forma seu } \\
\text { humor e níveis de estresse e } 33 \% \text { relataram que } \\
\text { seu consumo de substâncias como álcool e } \\
\text { cigarro foi alterado devido à pandemia. }\end{array}$ & $\begin{array}{l}\text { Social Science \& } \\
\quad \text { Medicine }\end{array}$ & Emery et al., 2021 \\
\hline $\begin{array}{l}\text { O estresse e a saúde mental de } \\
\text { profissionais da linha de frente da } \\
\text { COVID-19 em hospital geral }\end{array}$ & $\begin{array}{c}\text { De } 123 \text { profissionais entrevistados, } 13 \% \\
\text { utilizavam algum psicofármaco no momento. } \\
\text { Também foi relatado o uso eventual de álcool } \\
\text { por } 41 \% \text { e tabaco por } 10 \% .\end{array}$ & $\begin{array}{l}\text { Jornal Brasileiro de } \\
\text { psiquiatria }\end{array}$ & Horta et al., 2021 \\
\hline $\begin{array}{l}\text { Mudanças no uso de álcool em função } \\
\text { de sofrimento psicológico e suporte } \\
\text { social após o fechamento de } \\
\text { universidades relacionadas ao COVID- } \\
19\end{array}$ & $\begin{array}{l}\text { Estudo com } 1958 \text { alunos, mostrou aumento no } \\
\text { consumo de álcool durante a pandemia, } \\
\text { principalmente entre os que já possuíam } \\
\text { histórico de depressão e ansiedade. }\end{array}$ & Addict Behav. & $\begin{array}{l}\text { Lechner et al., } \\
\qquad 2020\end{array}$ \\
\hline $\begin{array}{l}\text { Distanciamento social, sentimento de } \\
\text { tristeza e estilos de vida da população } \\
\text { brasileira durante a pandemia de } \\
\text { COVID-19 }\end{array}$ & $\begin{array}{l}\text { Foram encontrados dados frequentes sobre } \\
\text { sentimento e tristeza e depressão ( } 35,5 \%) \text {, } \\
\text { isolamento e ansiedade ( } 41,2 \% \text { e } 41,3 \% \\
\text { respectivamente). } 17 \% \text { dos entrevistados } \\
\text { relataram aumento no consumo de bebidas } \\
\text { alcoólicas. }\end{array}$ & Saúde em debate & Malta et al., 2020 \\
\hline $\begin{array}{l}\text { Fatores psicossociais e Síndrome de } \\
\text { Burnout entre os profissionais dos } \\
\text { serviços de saúde mental }\end{array}$ & $\begin{array}{c}\text { Prevalência de Síndrome de Burnout em } \\
\text { profissionais da enfermagem, tendo associado a } \\
\text { alta jornada de trabalho com aumento do uso } \\
\text { de psicofármacos. }\end{array}$ & $\begin{array}{l}\text { Revista latino- } \\
\text { Americana de } \\
\text { Enfermagem }\end{array}$ & $\begin{array}{l}\text { Moreira \& Lucca, } \\
2020\end{array}$ \\
\hline $\begin{array}{l}\text { Influência da mídia sobre a população: } \\
\text { estudo de caso sobre os medicamentos } \\
\text { mais vendidos durante a pandemia de } \\
\text { covid-19 em três municípios da região } \\
\text { norte de Mato Grosso. }\end{array}$ & $\begin{array}{l}\text { O estudo aponta para um aumento na venda de } \\
\text { medicamentos ansiolíticos e antidepressivos }\end{array}$ & $\begin{array}{l}\text { IV Congresso } \\
\text { Brasieleiro de } \\
\text { Ciências da Saúde }\end{array}$ & Rufino et al., 2020 \\
\hline
\end{tabular}

\section{Discussão}

\section{Saúde mental em meio a pandemia da SARS-CoV-2}

A COVID-19 causou danos irreparáveis ao redor do mundo, tendo impactos não somente na saúde física de indivíduos, como também na saúde mental da população. Os impactos da pandemia da SARS-CoV-2 principalmente no que diz respeito a medidas de distanciamento e isolamento social, provocaram um aumento de transtornos mentais e o surgimento de diversas patologias. No estudo realizado por Huang \& Zhao (2020), com 7.236 voluntários chineses, a respeito de aparecimento de Transtorno de Ansiedade Generalizado (TAG), sintomas depressivos, estresse e baixa qualidade de sono, indicaram o aumento no aparecimento ou agravamento destas patologias, sendo mais frequente o TAG e sintomas depressivos em jovens ( $<35$ anos) com uso maior do que 3 horas consumindo informações sobre a COVID-19 e profissionais de saúde, principalmente os de linha de frente, associado a baixa qualidade de sono e intensa preocupação com contaminação própria e de familiares.

Já, no estudo de Horta et al. (2021) foi evidenciado do total de 123 profissionais que trabalhavam na linha de frente enfrentamento da COVID-19, 41\% dos entrevistados possuíam indicativo de Burnout, mediante aplicação do Inventário de Burnout Oldenburg (OBI). Em outro estudo, Moreira e Lucca (2020) também mostram um crescente número de indivíduos, principalmente profissionais de saúde e trabalhadores essenciais com altas pontuações em inventário de Burnout.

Barros et al. (2020), evidenciam em um estudo realizado com 45.161 pacientes de diferentes regiões do Brasil entre os meses de abril e maio de 2020 , onde $53,6 \%$ os participantes da amostra eram do sexo feminino, 
20,3\% contavam 60 anos ou mais, e 24,7\% 18 a 29 anos de idade. 14,9\% registravam antecedente/diagnóstico prévio de depressão. Entre os adultos jovens (18 a 29 anos), 44,0\% eram estudantes, e 19,2\% casados, mostrando que durante o início da pandemia, houve um aumento da prevalência de diagnósticos de depressão ou ansiedade de aproximadamente $50 \%$ da população brasileira, tendo afetado de forma mais expressiva adultos jovens na faixa entre 18-29 anos.

Corroborando com este resultado, Feter et al. (2021), em seu estudo sobre depressão e ansiedade durante o período de isolamento social entre junho e julho de 2020, apontam para um aumento de 6,6 vezes no diagnóstico de depressão e 7,4 vezes da ansiedade, sendo que a maior proporção foi em mulheres na faixa entre 18-30 anos, principalmente aquelas que já possuíam algum tipo de doença crônica pré-existente ou que tiveram seus rendimentos influenciados negativamente pela pandemia. Corroborando com essas informações, Silva (2020) e Duarte et al. (2020) associam o estresse frente à pandemia de COVID-19 ao desenvolvimento de depressão principalmente em mulheres em idade jovem.

Complementando este dado, Santos et al. (2021) associam o fato de haver maior índice de mulheres trabalhando no setor da saúde, possibilitando que essas mulheres atuem na linha de frente, gerando desta forma o agravamento no quadro de estresse e, respectivamente, depressão. Segundo Sousa et al. (2020), os sentimentos negativos e relacionados à ansiedade também estão extremamente presentes nos discursos de homens, principalmente àqueles que se encontram de alguma forma afetados emocional e/ou financeiramente pelo isolamento e pandemia.

\section{Uso de substâncias psicotrópicas durante a pandemia}

De acordo com o United Nations Office on Drug and Crime (UNODC), até o ano de 2018, a Cannabis seria a substância ilícita mais consumida, porém, com menor risco de transtornos associados se comparado a outras substâncias, como os opióides. Ainda de acordo com o UNODC mais de 35 milhões de pessoas ao redor do mundo desenvolveram algum tipo de transtorno associado ao uso de drogas até o ano de 2018.

Com o surgimento da COVID-19, a tendência para o consumo de substâncias foi alterada, gerando maior escassez de opióides e o aumento no uso de outras substâncias de mais fácil acesso, como álcool e benzodiazepínicos (UNODC, 2020).

Rufino et al. (2020) em estudo conduzido em farmácias do Mato Grosso, mostram um aumento de $40 \%$ na venda de medicamentos como a Fluoxetina e Alprazolam entre os meses de março e agosto de 2020, além do aumento da venda de fitoterápicos destinados no controle dos sintomas da ansiedade e depressão, gerando uma correlação com aumento não apenas de indivíduos devidamente diagnosticados com transtornos como depressão e ansiedade por profissionais, mas também alertando para um aumento da automedicação com substâncias que não necessitam de receita médica, como os fitoterápicos.

Além do aumento do uso indiscriminado de medicamentos para ansiedade e depressão, o consumo de outras substâncias aumentou durante a pandemia. Segundo Czeisler et al. (2020), 40\% dos adultos da população Norte Americana relataram algum tipo de dificuldade com relação à manutenção da saúde mental durante os meses de junho e julho de 2020. Por sua vez, 13\% destes relataram início ou aumento no consumo de substâncias psicotrópicas. Emery et al. (2021) também apontam o aumento do uso de psicotrópicos por $33 \%$ dos indivíduos presentes em seu estudo realizado no mesmo país do autor anterior.

Dumas et al. (2020) apontam em estudo realizado com aproximadamente 1.316 adolescentes canadenses entre 14-18 anos, um aumento no consumo de substâncias como Cannabis e uso de vapers. Almandoz et al. (2021), verificaram que do total de 589 indivíduos com diagnóstico de obesidade, aproximadamente 10\% faziam uso de sedativos, 3,6\% de cannabis e $1 \%$ de estimulantes, relacionando o aumento no consumo destas substâncias devido à piora na saúde mental em meio ao período de isolamento social.

O consumo elevado de álcool também é frequentemente relatado em meio a população que apresentam sentimentos de tristeza, isolamento e depressão. Principalmente dentre os que cumpriram o isolamento social. Lechner et al. (2020) apontam maior consumo de álcool entre indivíduos com sintomas associados a transtornos mentais pré-pandêmicos. Ahmed et al. (2020), em seu estudo conduzido com mais 1000 chineses, ressaltam para um maior consumo de álcool entre a população na faixa entre 21 e 40 anos. Malta et al. (2020) aponta em seu estudo transversal realizado entre os meses de abril e maio de 2020, um aumento a sentimentos de tristeza ou depressão, bem como aumento de consumo de bebidas alcoólicas em $17 \%$ e de cigarros entre pessoas previamente fumantes, de até $34 \%$ no período de isolamento social.

Werneck et al. (2020) alertam não somente para o aumento do consumo de diversas substâncias psicotrópicas como álcool e cigarro, como também realiza uma correlação entre indivíduos mais ansiosos e a 
aquisição de piores hábitos alimentares, com aumento de consumo de alimentos ricos em sal ou açúcar, provocando mais danos à saúde, a longo prazo.

Desta forma, os resultados apontam para um aumento não apenas no uso de fármacos, mas também de álcool, substâncias ilícitas e hábitos alimentares prejudiciais à saúde como forma de minimizar efeitos de possíveis transtornos causados pela Pandemia de COVID-19.

\section{Possíveis formas de enfrentamento ao uso de psicotrópicos em meio a pandemia}

Diversos autores apontam a prática de autocuidado, realização de psicoterapia, o uso controlado de redes sociais como forma de informação, possuir uma rede de apoio familiar ou de amigos e a prática de exercícios físicos como fatores importantes na redução do uso de psicotrópicos, mesmo quando em grupos com maior incidência de níveis de estresse, como profissionais de linha de frente.

Torres et al. (2020) apontam o apoio mútuo entre jovens adultos por meio de diálogos online como forma de minimizar os riscos trazidos pelo medo causado devido ao isolamento social.

Rufino et al. (2020), bem como Bendau et al. (2021), evidenciam que um alto consumo de grandes mídias, bem como redes sociais e TV tendem a causar um efeito de "infodemia", provocando aumento da incidência de depressão e ansiedade, sendo necessário haver um consumo moderado de informações por meio de mídias sociais.

Estudos como Werneck et al. (2020), apontam também a associação entre indivíduos que possuem alimentação saudável e praticantes de atividades físicas e menores riscos de desenvolvimento de hábitos prejudiciais durante o quadro de depressão e ansiedade durante os períodos de isolamento social dentro da pandemia de COVID-19.

\section{Considerações finais}

Além do medo de contrair a doença, a pandemia do novo Coronavírus tem provocado em diversas pessoas um sentimento intenso de insegurança não apenas sobre a saúde, mas também nas esferas social e econômica. Este sentimento, por sua vez, pode desencadear o aumento no consumo de medicamentos específicos para tratamento de ansiedade e depressão, além de substâncias como álcool, tabaco, drogas ilícitas, além de aumento em hábitos menos saudáveis relacionados à alimentação.

Dentro dos grupos mais afetados, evidenciamos uma crescente instabilidade na saúde mental e aquisição de piores hábitos de vida principalmente por mulheres jovens e profissionais de saúde.

O presente estudo aponta para um aumento significativo do uso de fármacos psicotrópicos entre jovens e adultos, em meio a pandemia da SARS-CoV-2, reforçando o uso indiscriminado de medicamentos e outras substâncias, lícitas ou ilícitas como forma de minimizar os efeitos psicológicos causados pela pandemia, principalmente no ano de 2020.

Entretanto, por se tratar de um tema relativamente novo, destaca-se a necessidade de verificar mais a fundo a população brasileira e propor estudos que identifiquem os mecanismos, sobretudo farmacológicos, para enfrentamento destes transtornos, em especial, entre os jovens universitários devido a imaturidade e interrupção dos projetos pessoais.

\section{Referências}

Ahmed, M. Z., Ahmed, O., Aibao, Z., Hanbin, S., Siyu, L., \& Ahmad, A. 2020. Epidemic of COVID-19 in China and associated Psychological Problems. Asian Journal of Psychiatry, 51, 102092.

Almandoz, J. P., Xie, L., Schellinger, J. N., Mathew, M. S., Bismar, N., ..., \& Messiah, D. V. S. E. 2021. Substance use, mental health and weight-related behaviours during the COVID-19 pandemic in people with obesity. Clinical Obesity, 11(2), e12440.

Bahia. 2020. Decreto $n^{\circ} 32.357$ de 20 de Abril de 2020. Define medidas complementares para enfrentamento da pandemia decorrente do coronavírus.

Barros, M. B. A., Lima, M. G., Malta, D. C., Szwarcwald, C. L., ..., \& Gracie, R. 2020. Relato de tristeza/depressão, nervosismo/ansiedade e problemas de sono na população adulta brasileira durante a pandemia de COVID-19. Epidemiologia e Serviços de Saúde, 29(4), e2020427.

Bendau, A., Petzold, M. B., Pyrkosch, L., Mascarell Maricic, L., Betzler, F., Rogoll, J., Große, J., Ströhle, A., \& Plag, J. 2021. Associations between COVID-19 related media consumption and symptoms of anxiety, 
depression and COVID-19 related fear in the general population in Germany. European Archives of Psychiatry and Clinical Neuroscience, 271(2), 283-291.

Caetano, R., Silva, A. B., Guedes, A. C. C. M., Paiva, C. C. N., ... \& Silva, R. M. Desafios e oportunidades para telessaúde em tempos da pandemia pela COVID-19: uma reflexão sobre os espaços e iniciativas no contexto brasileiro. 2020. Cadernos de Saúde Pública, 36(5), e00088920.

CDC COVID-19 Response Team. 2020. Severe outcomes among patients with Coronavirus Disease 2019 (COVID-19). Morbidity and Mortality Weekly Report (MMWR), 69(12), 343-346.

Czeisler M.É., Lane, R. I., Petrosky, E., Wiley, J. F., ... \& Rajaratnam, S. M. W. 2020. Mental Health, Substance Use, and Suicidal Ideation During the COVID-19 Pandemic - United States, June 24-30, 2020. Morbidity and Mortality Weekly Report (MMWR), 69(32), 1049-1057.

Duarte, M. Q., Silva Santo, M. A., Lima, C. P., Giordani, J. P., \& Trentini, C. M. 2020. COVID-19 e os impactos na saúde mental: uma amostra do Rio Grande do Sul, Brasil. Ciência \& Saúde Coletiva, 25(9), 3401-3411.

Dumas T. M., Ellis, W., Litt, D. M. 2020. What Does Adolescent Substance Use Look Like During the COVID19 Pandemic? Examining Changes in Frequency, Social Contexts, and Pandemic-Related Predictors. Journal of Adolescent Health, 67, 354-361.

Emery, R. L., Johnson, S. T., Simone, M., Loth, K. A., Berge, J. M., \& Neumark-Sztainer, D. 2021. Understanding the impact of the COVID-19 pandemic on stress, mood, and substance use among young adults in the greater Minneapolis-St. Paul area: Findings from project EAT. Social Science \& Medicine, 276, 113826.

Faro, A., Bahiane, M. A., Nakano, T. C., Reis, C., Silva, B. F. P., \& Vitti, L. S. 2020. COVID-19 e saúde mental: a emergência do cuidado. Estududos de Psicologia (Campinas), 37, e200074.

Feter, N., Caputo, E. L., Doring, I. R., Leite, J. S., Cassuriaga, J., Reichert, F. F., da Silva, M. C., Coombes, J. S., \& Rombaldi, A. J. 2021. Sharp increase in depression and anxiety among Brazilian adults during the COVID-19 pandemic: findings from the PAMPA cohort. Public Health, 190, 101-107.

Fontes, A. P., \& Neri A. L. 2015. Resilience in Aging: Literature Review. Ciências \& Saúde Coletiva, 20(5), 1475-95.

Horta, R. L, Camargo, E. G., Barbosa, M. L. L., Lantin, P. J. S., ..., \& Lutzky, B. A. 2021. O estresse e a saúde mental de profissionais da linha de frente da COVID-19 em hospital geral. Jornal Brasileiro de Psiquiatria, 70(1), 30-38.

Huang, Y., \& Zhao, N. 2020. Generalized anxiety disorder, depressive symptoms and sleep quality during COVID-19 outbreak in China: a web-based cross-sectional survey. Psychiatry Research, 288, 112954.

Lechner, W. V., Laurene, K. R., Patel, S., Anderson, M., Grega, C., \& Kenne, D. R. 2020. Changes in alcohol use as a function of psychological distress and social support following COVID-19 related University closings. Addictive behaviors, 110, 106527.

Malta, D. C., Gomes, C. S., Szwarcwald, C. L., Barros, M. B. A., ... \& Silva, D. R. P. 2020. Distanciamento social, sentimento de tristeza e estilos de vida da população brasileira durante a pandemia de COVID-19. Saúde em Debate, 44(4), 177-190.

Moreira, A. S., \& Lucca, S. R. 2020. Fatores psicossociais e Síndrome de Burnout entre os profissionais dos serviços de saúde mental. 2020. Revista Latino-Americana de Enfermagem, 28, e3336.

Rufino P. S., Reginatto, E. L., Dias, V. H. H., \& Santos, M F. 2020. Influência da mídia sobre a população: estudo de caso sobre os medicamentos mais vendidos durante a pandemia de Covid-19 em três municípios da região norte de Mato Grosso. In: Anais IV CONBRACIS - Congresso Brasileiro de Ciências da Saúde, Campina Grande: Realize Editor.

Silva, H. G. N., Santos, L. E. S., \& Oliveira, A. K. S. 2020. Efeitos da pandemia no novo Coronavírus na saúde mental de indivíduos e coletividades. Journal of Nursing and Health, 10(4), 20104007.

Silva, W. A. D. 2020. Covid-19 no Brasil: estresse como preditor da depressão. Scielo Preprint. Disponível em: <https://preprints.scielo.org/index.php/scielo/preprint/view/1132/1691>. Acesso em: 25 de março de 2021.

Santos, K. M., Galvão, M. H. R., Gomes, S. M., Souza, T. A., Medeiros, A. A., \& Barbosa, I. R. 2021. Depressão e ansiedade em profissionais de enfermagem durante a pandemia da covid-19. Escola Anna Nery, 25, e20200370.

Sousa, R. A., Carvalho, E. S. S., Santana, T. S., Sousa, A. F. L., ... \& Pereira, A. 2020. Sentimento e emoções de homens no enquadramento da doença Covid-19. 2020. Ciência \& Saúde Coletiva, 25(9), 3481-3491.

Torres, R. A. M., Veras, K. C. B. B., Torres, J. D. M., Gomes, E. D. P., Martins, J. V., \& Soeiro, A. C. C. 2020. Saúde mental das juventudes e COVID-19: discursos produtores do webcuidado educativo mediados na webrádio. Nursing (São Paulo), 23(270), 4887-4896. 
UNODC. United Nations Office on Drug and Crime. 2020. Relatório Mundial sobre Drogas 2020: consumo global de drogas aumenta, enquanto COVID-19 impacta mercados, aponta relatório. Disponível em: <https://www.unodc.org/lpo-brazil/pt/frontpage/2020/06/relatrio-mundial-sobre-drogas-2020_consumo-global-de-drogas-aumenta--enquanto-covid-19-impacta-mercado.html>.

Velavan, T. P., \& Meyer, C. G. 2020. The COVID-19 epidemic. Tropical Medicine \& International Health, 25(3), 278-280.

Werneck, A. O., Silva, D. R., Malta, D. C., Souza-Júnior, P. R. B., ..., \& Szwarcwald, C. L. 2020. Lifestyle behaviors changes during the COVID-19 pandemic quarantine among 6,881 Brazilian adults with depression and 35,143 without depression. Ciência \& Saúde Coletiva, 25(Supl. 2), 4151-4156.

WHO. World Health Organization. 2020. Coronavirus Disease (COVID-2019) situation reports. Disponível em: <https://www.who.int/emergencies/diseases/novel-coronavirus-2019/situation-reports>. Acesso em : 9 de junho de 2020.

\section{Minicurrículo}

Fernanda Pinto Dantas. Graduanda do curso de Farmácia, da Universidade Salvador (Unifacs), Salvador, Ba, Brasil.

Fernando Maia Pereira Santos. Graduando do curso de Farmácia, da Universidade Salvador (Unifacs), Salvador, Ba, Brasil.

Bruna Dallaqua. Farmacêutica graduada pela Universidade do Sagrado Coração - USC em 2007. Mestre e doutora em Ginecologia, Obstetrícia e Mastologia com ênfase em farmácia pela Faculdade de Medicina de Botucatu - FMB - Unesp. Atualmente é professora da Universidade Salvador (Unifacs) e do Centro Universitário UniRuy Barbosa, nesta última atua também como preceptora líder dos estágios curriculares do curso de farmácia. Tem experiência em pesquisa na área de Bioquímica, Fisiologia e Farmacologia com ênfase nas repercussões materno-fetais causadas pelo Diabete mellitus.

Como citar: Oliveira, F.P.D., Santos, F.M.P., \& Dallaqua, B. 2021. Consumo de psicotrópicos em meio a pandemia do Sars-CoV-2. Pubsaúde, 7, a187. DOI: https://dx.doi.org/10.31533/pubsaude7.a187

Recebido: 13 mai. 2021.

Revisado e aceito: 31 mai. 2021.

Conflito de interesse: os autores declaram, em relação aos produtos e companhias descritos nesse artigo, não ter interesses associativos, comerciais, de propriedade ou financeiros que representem conflito de interesse.

Licenciamento: Este artigo é publicado na modalidade Acesso Aberto sob a licença Creative Commons Atribuição 4.0 (CC-BY 4.0). 